\title{
Preoperative Prognostic Nutritional Index Predicts Long-term Outcome in Gastric Cancer: A Propensity Score-matched Analysis
}

\author{
NORIYUKI HIRAHARA, YOSHITSUGU TAJIMA, YUSUKE FUJII, TETSU YAMAMOTO, \\ RYOJI HYAKUDOMI, TAKAHITO TANIURA, SHUNSUKE KAJI and YASUNARI KAWABATA \\ Department of Digestive and General Surgery, Shimane University Faculty of Medicine, Shimane, Japan
}

\begin{abstract}
Background/Aim: Recent evidence suggests that preoperative malnutrition may lead to poor survival in cancer patients. This study aimed to determine the ability of the prognostic nutritional index (PNI) to predict survival in gastric cancer patients. Patients and Methods: Two hundred and eighteen patients who had undergone laparoscopic gastrectomy were retrospectively reviewed via propensity score-matched analysis. Results: In multivariate analysis of overall patients, pTNM stage, carcinoembryonic antigen, and PNI were independent predictors of overall survival (OS), and pTNM stage and PNI were independent predictors of cancer-specific survival (CSS). Among the 92 non-elderly patients, pTNM stage and PNI were independent predictors of $O S$, and pTNM stage, PNI, and adjuvant chemotherapy were independent predictors of CSS in multivariate analysis. On the other hand, among the 126 elderly patients, low PNI value was identified as a significant predictor of shorter OS in univariate analysis. Conclusion: PNI is associated with OS and CSS in gastric cancer patients, especially nonelderly patients.
\end{abstract}

The tumor-node-metastasis (TNM) staging system is known to be a significant parameter for predicting the clinical course of patients with cancer (1). Despite its widespread use, the TNM classification merely focuses on tumor extension, irrespective of the biological behavior of the tumor or the host antitumor response. Recent studies reported that a preoperative malnutrition and immunosuppressive status can

Correspondence to: Noriyuki Hirahara, MD, Ph.D., Department of Digestive and General Surgery, Shimane University Faculty of Medicine, 89-1 Enya-cho, Izumo, Shimane 693-8501, Japan. Tel: +81853202232, Fax: +81853202229, e-mail: norinorihirahara@ yahoo.co.jp

Key Words: Prognostic nutritional index, gastric cancer, overall survival, cancer-specific survival. lead to poor survival in patients with cancer $(2,3)$. Several scoring tools for assessing preoperative nutritional status are now available. The prognostic nutritional index (PNI), which is based on the level of lymphocytes and albuminemia, is a useful parameter to indicate the immune and nutritional status of patients with different types of malignant tumors (4-7). The PNI is associated with the prognosis of patients with cancer because serum albumin is a potential indicator not only of the nutrition and systemic inflammatory response, but also of the antitumor immune response, and lymphocytes play a crucial role in the host cellular adaptive immune response (8). Therefore, the PNI can be considered a good indicator of prognosis, as well as, the immune and nutritional status in patients with cancer.

In this study, propensity score-matched (PSM) analysis was used to determine the ability of the PNI to predict survival, especially age-stratified long-term survival, in patients with gastric cancer.

\section{Patients and Methods}

Patients. A database of medical records from 449 consecutive patients who had undergone gastrectomy for gastric cancer at our institution between January 2009 and December 2016 was retrospectively reviewed. The inclusion criteria were as follows: 1) age 20-89 years, 2) histologically confirmed gastric adenocarcinoma, 3) no distant metastasis, 4) an Eastern Cooperative Oncology Group performance status of 0-2, and 5) curative gastrectomy with R0 resection. Patients were excluded if they had T4b disease; remnant gastric cancer; an emergency gastrectomy for bleeding or perforation; post-neoadjuvant chemotherapy; and hematological, inflammatory, or autoimmune disorders. In total, 368 patients were enrolled in the study (Figure 1).

All patients underwent laparoscopic or laparoscopy-assisted gastrectomy. The extent of gastrectomy and lymph node dissection was determined according to the Japanese gastric cancer treatment guidelines (version 3) (9). Five-fluorouracil-based adjuvant chemotherapy regimens (cisplatin plus tegafur/gimeracil/oteracil or capecitabine) were recommended to the majority of patients with advanced gastric cancer. 


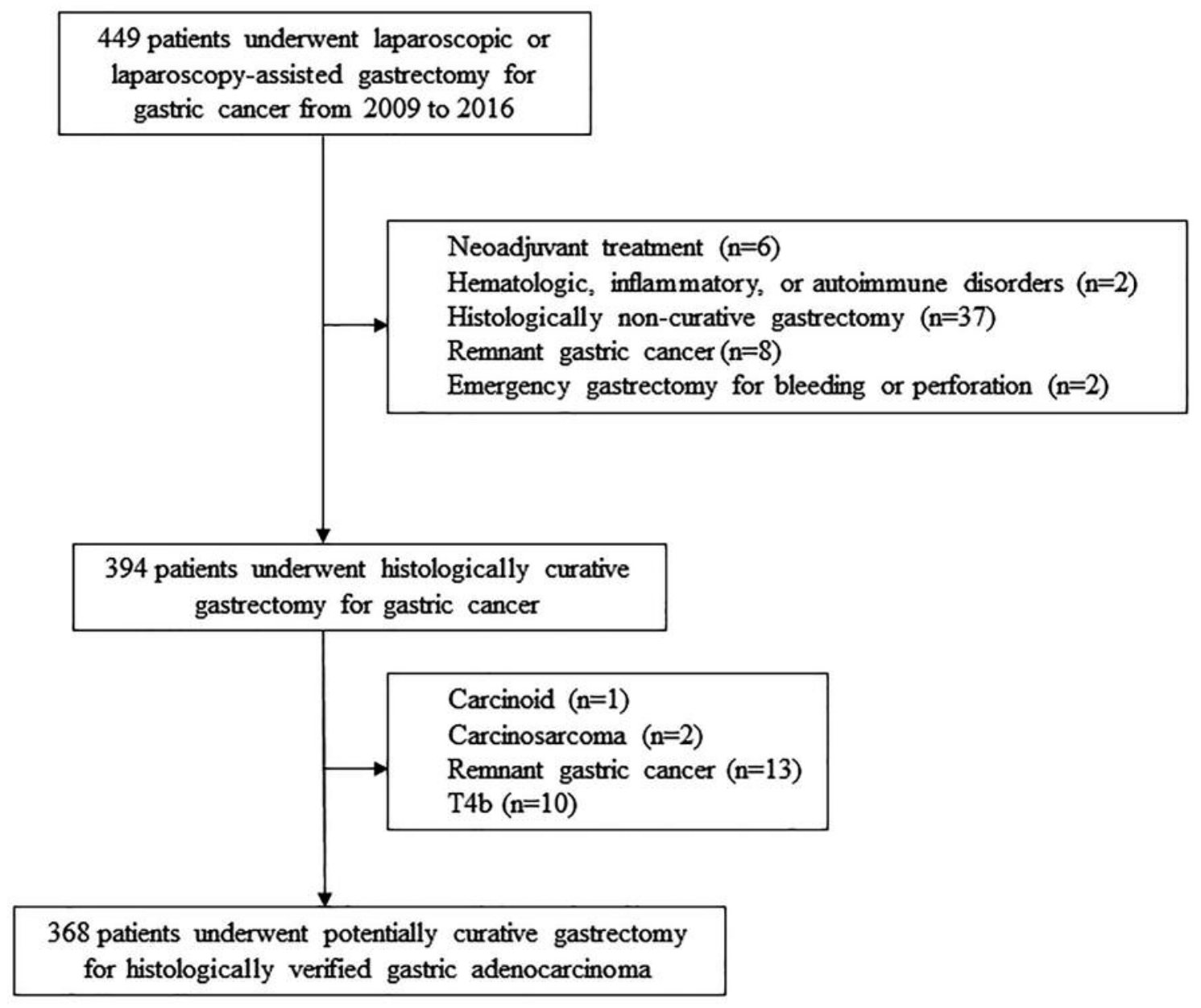

Figure 1. Study flow diagram.

The patients' clinical characteristics, laboratory data, treatment data, and pathological data were collected from reviewing medical records. Tumor stage was determined according to the American Joint Committee on Cancer TNM staging system (7th edition) (1).

The observation period was from the date of surgery until the date of death, loss to follow-up, or withdrawal of consent. Overall survival (OS) and cancer-specific survival (CSS) were evaluated following gastrectomy. OS was calculated from the date of primary gastrectomy to the date of death from any cause or last follow-up. CSS was calculated from the date of primary gastrectomy to the date of cancer-specific death or withdrawal of consent.

Patients were stratified into two groups according to age: $<70$ years (non-elderly) and $\geq 70$ years (elderly).

Preoperative nutritional parameters. All laboratory data used for calculating preoperative nutritional parameters were obtained within 1 week before surgery. The following items were selected as concise constitutional evaluation methods: body mass index (BMI) (body weight $[\mathrm{kg}] /$ height $[\mathrm{m} 2])$ and the PNI $(10 \times$ serum albumin $[\mathrm{g} / \mathrm{dl}]+0.005 \times$ total lymphocyte count in peripheral blood $\left.\left[/ \mathrm{mm}^{3}\right]\right)(4)$.
A receiver operating characteristic (ROC) curve of the preoperative PNI was generated for the multiple logistic regression analysis of OS and CSS. The area under the ROC curve (AUC) estimation method was used to assess the ability of the preoperative PNI to predict OS and CSS. The optimal cut-off value for the preoperative PNI was set at 44.3 in this study, based on OS (sensitivity, 77.8\%; specificity, 56.3\%; AUC=0.681) and CSS (sensitivity, 73.2\%; specificity, 52.5\%; AUC=0.592). Patients were categorized as having a low $(<44.3)$ or high PNI value $(\geq 44.3)$ (Figure 2a-b).

Ethics approval and consent to participate. This study was approved by the Ethical Review Board of Shimane University Faculty of Medicine (Shimane, Japan). Research was conducted in accordance with the 1964 Declaration of Helsinki and its later amendments. The requirement for informed consent was waived, due to the retrospective nature of this study.

Statistical analyses. Continuous variables were expressed as the mean \pm standard deviation or median and range. Comparisons were performed using the Chi-square test or Student's $t$-test for 
(a)

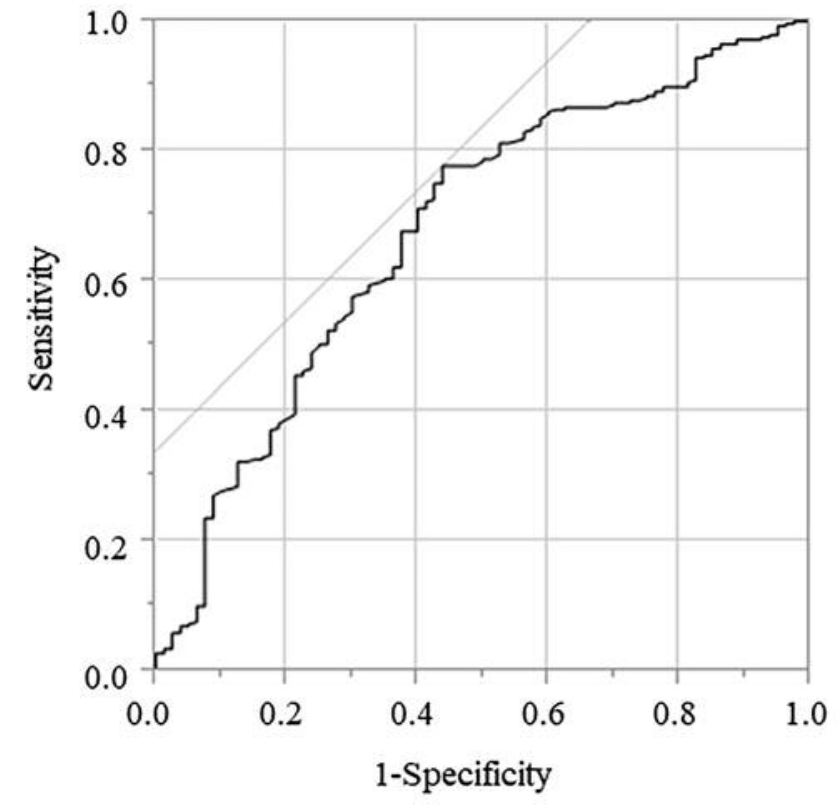

(b)

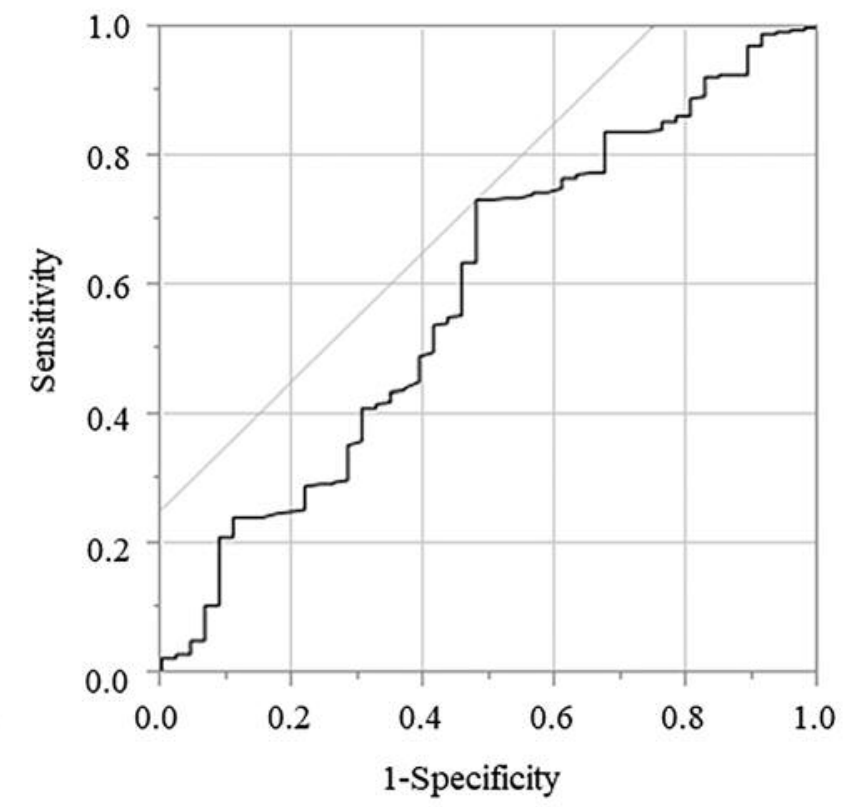

Figure 2. Receiver operating characteristic curves for (a) overall survival and (b) cancer-specific survival. Receiver operating characteristic curves were plotted to determine the optimal cutoff value for the prognostic nutritional index.

categorical variables and the Mann-Whitney $U$-test for nonnormally distributed continuous variables. OS and CSS were calculated using the Kaplan-Meier method and compared by the log-rank test. Univariate analysis was performed to identify factors associated with OS and CSS. Variables with a $p<0.05$ in the univariate analysis were included in the multivariate analysis using a Cox proportional hazards model to determine the independent prognostic factors. Potential prognostic factors included age $(<70 v s . \geq 70$ years), sex (female $v s$. male), BMI $\left(<18.5 v s . \geq 18.5 \mathrm{~kg} / \mathrm{m}^{2}\right)$, tumor size $(<5.0 v s . \geq 5.0 \mathrm{~cm})$, cancer cell differentiation (well- $v s$. moderately/poorly differentiated), pathological TNM (pTNM) stage (Stage I, II $v s$. III), preoperative serum carcinoembryonic antigen (CEA) level $(<5.0 \mathrm{vs} . \geq 5.0$ $\mathrm{ng} / \mathrm{ml})$, the PNI (<44.3 vs. $\geq 44.3)$, and postoperative adjuvant chemotherapy ("no" vs. "yes").

All statistical analyses were conducted using JMP software for Windows (version 11; SAS Institute, Cary, NC, USA). A two-sided $p<0.05$ was considered statistically significant. To reduce bias due to different distributions of covariables between groups of patients with a low and high PNI, PSM analysis was performed using R statistical package (version 3.1.3) (http://www.r-project.org). The following covariables were matched: tumor depth, lymph node metastasis, and pTNM stage.

\section{Results}

Clinicopathological characteristics of overall patients with gastric cancer. The associations between the PNI and the clinicopathological characteristics of the entire cohort of
368 patients with gastric adenocarcinoma are summarized in Table I. Based on a PNI cutoff value of 44.3, 109 patients $(29.6 \%)$ were classified as having a low PNI and the remaining 259 patients $(70.4 \%)$ were classified as having a high PNI. PNI values were significantly associated with age $(p<0.001)$, BMI $(p<0.001)$, white blood cell count $(p=0.004)$, red blood cell count $(p<0.001)$, tumor size $(p<0.001)$, tumor depth $(p<0.001)$, lymph node metastasis $(p=0.010)$, pTNM stage $(p<0.001)$, intraoperative bleeding $(p=0.029)$, serum albumin concentration $(p<0.001)$, and $\mathrm{C}$-reactive protein (CRP) level $(p<0.001)$.

Clinicopathological characteristics of propensity score matched patients with gastric cancer. After applying PSM analysis, the distributions of confounding variables (tumor depth, lymph node metastasis, and pTNM stage) were adequately balanced between the low and high PNI groups. This allowed us to generate 109 matched pairs with a standardized or individualized follow-up, which were used in subsequent analyses as indicated. PNI values were significantly associated with age $(p<0.001)$, BMI $(p=0.012)$, white blood cell count $(p=0.002)$, red blood cell count $(p<0.001)$, tumor size $(p=0.001)$, serum albumin concentration $(p<0.001)$, CRP level $(p<0.001)$, and adjuvant chemotherapy $(p=0.035)$ (Table II). 
Table I. Baseline characteristics of PNI stratified overall patients.

\begin{tabular}{|c|c|c|c|c|}
\hline \multirow[t]{2}{*}{ Characteristics } & \multirow{2}{*}{$\begin{array}{c}\text { Total } \\
\text { patients }\end{array}$} & \multicolumn{2}{|c|}{ PNI } & \multirow[b]{2}{*}{$p$-Value } \\
\hline & & $<44.3(\mathrm{n}=109)$ & $\geq 44.3(n=259)$ & \\
\hline Age (years) & & $78(46-91)$ & $69(36-89)$ & $<0.001$ \\
\hline Gender & & & & 0.124 \\
\hline Male & 254 & 69 & 185 & \\
\hline Female & 114 & 40 & 74 & \\
\hline BMI & & $21.4(13.9-30.7)$ & $22.6(16.5-32.8)$ & $<0.001$ \\
\hline WBC $(\mu \mathrm{l})$ & & $5320(1830-9750)$ & $5760(510-10300)$ & 0.004 \\
\hline $\mathrm{RBC}\left(\times 10^{4} \mu \mathrm{l}\right)$ & & $366(223-520)$ & $439(203-579)$ & $<0.001$ \\
\hline Location of tumor & & & & 0.187 \\
\hline EGJ & 11 & 2 & 9 & \\
\hline $\mathrm{U}$ & 70 & 23 & 47 & \\
\hline M & 162 & 40 & 122 & \\
\hline $\mathrm{L}$ & 125 & 44 & 81 & \\
\hline Tumor size $(\mathrm{mm})$ & & $55(7-170)$ & $36(3-180)$ & $<0.001$ \\
\hline Procedure & & & & 0.090 \\
\hline LTG & 82 & 31 & 51 & \\
\hline LPG & 37 & 7 & 30 & \\
\hline L(A)DG & 249 & 71 & 178 & \\
\hline Differentiation & & & & 0.123 \\
\hline Well & 71 & 14 & 57 & \\
\hline Moderate & 134 & 42 & 92 & \\
\hline Poor & 163 & 53 & 110 & \\
\hline Depth of tumor & & & & $<0.001$ \\
\hline T1a-1b & 190 & 40 & 150 & \\
\hline 2 & 48 & 12 & 36 & \\
\hline 3 & 54 & 23 & 31 & \\
\hline $4 a-4 b$ & 74 & 34 & 40 & \\
\hline Lymph node metastasis & & & 0.010 & \\
\hline NO & 244 & 59 & 185 & \\
\hline N1 & 40 & 14 & 26 & \\
\hline N2 & 42 & 19 & 23 & \\
\hline N3 & 42 & 17 & 25 & \\
\hline pTNM stage & & & & $<0.001$ \\
\hline $\mathrm{Ia}-\mathrm{Ib}$ & 217 & 45 & 172 & \\
\hline IIa-IIb & 65 & 26 & 39 & \\
\hline IIIa-IIIc & 86 & 38 & 48 & \\
\hline Operation time (min) & & $399(177-911)$ & $398(218-836)$ & 0.542 \\
\hline Intraoperative bleeding $(\mathrm{ml})$ & & $100(0-5850)$ & $20(0-3600)$ & 0.029 \\
\hline Albumin $(\mathrm{g} / \mathrm{dl})$ & & $3.4(1.5-4.0)$ & $4.2(3.5-5.1)$ & $<0.001$ \\
\hline $\mathrm{CRP}(\mathrm{mg} / \mathrm{l})$ & & $0.22(0.01-7.09)$ & $0.06(0.002-6.31)$ & $<0.001$ \\
\hline $\mathrm{CEA}(\mathrm{ng} / \mathrm{ml})$ & & & & 0.117 \\
\hline$<5$ & 286 & 79 & 207 & \\
\hline$\geq 5$ & 82 & 30 & 52 & \\
\hline Adjuvant chemotherapy & & & 0.541 & \\
\hline Yes & 100 & 32 & 68 & \\
\hline No & 268 & 77 & 191 & \\
\hline
\end{tabular}

PNI: Prognostic nutritional index; BMI: body mass index; WBC: white blood cell; RBC: red blood cell; EGJ: esophagogastric junction; U: upper; M: middle; L: lower; Differentiation: tumor differentiation; LTG: laparoscopic total gastrectomy; LPG: laparoscopic proximal gastrectomy; L(A)DG: laparoscopic (assisted) distal gastrectomy; pTNM stage: pathological TNM stage; CRP: C-reactive protein; CEA: carcinoembryonic antigen.

Cox regression analysis of postoperative survival in the entire cohort of propensity score matched patients with gastric cancer. In the PSM dataset, univariate analysis identified advanced pTNM stage $(p<0.001)$, a high serum
CEA level ( $p=0.017)$, a low PNI value $(p<0.001)$, and a high CRP level $(p=0.034)$ as significant risk factors for shorter OS. In the multivariate analysis, pTNM stage (hazard ratio $[\mathrm{HR}]=2.959,95 \%$ confidence interval $[\mathrm{CI}]=1.793-5.004$; 
Table II. Baseline characteristics of PNI stratified propensity score matched overall patients.

\begin{tabular}{|c|c|c|c|c|}
\hline \multirow[t]{2}{*}{ Characteristics } & \multirow{2}{*}{$\begin{array}{c}\text { Total } \\
\text { patients }\end{array}$} & \multicolumn{2}{|c|}{ PNI } & \multirow[b]{2}{*}{$p$-Value } \\
\hline & & $<44.3(\mathrm{n}=109)$ & $\geq 44.3(n=109)$ & \\
\hline Age (years) & & $78(46-91)$ & $68(36-89)$ & $<0.001$ \\
\hline Gender & & & & 0.315 \\
\hline Male & 145 & 69 & 76 & \\
\hline Female & 77 & 40 & 33 & \\
\hline BMI & & $21.4(13.9-30.7)$ & $22.4(16.8-32.8)$ & 0.012 \\
\hline WBC $(\mu \mathrm{l})$ & & $5320(1830-9750)$ & $5890(3080-10300)$ & 0.002 \\
\hline $\mathrm{RBC}\left(\times 10^{4} \mu \mathrm{l}\right)$ & & $366(223-520)$ & $432(279-548)$ & $<0.001$ \\
\hline Location of tumor & & & & 0.333 \\
\hline EGJ & 6 & 2 & 4 & \\
\hline $\mathrm{U}$ & 41 & 23 & 18 & \\
\hline M & 91 & 40 & 51 & \\
\hline $\mathrm{L}$ & 80 & 44 & 36 & \\
\hline Tumor size $(\mathrm{mm})$ & & $55(7-170)$ & $40(5-180)$ & 0.001 \\
\hline Procedure & & & & 0.954 \\
\hline LTG & 60 & 31 & 29 & \\
\hline LPG & 14 & 7 & 7 & \\
\hline $\mathrm{L}(\mathrm{A}) \mathrm{DG}$ & 144 & 71 & 73 & \\
\hline Differentiation & & & & 0.919 \\
\hline Well & 27 & 14 & 13 & \\
\hline Moderate & 82 & 42 & 40 & \\
\hline Poor & 109 & 53 & 56 & \\
\hline Depth of tumor & & & & 0.937 \\
\hline T1a-1b & 80 & 40 & 40 & \\
\hline 2 & 27 & 12 & 15 & \\
\hline 3 & 45 & 23 & 22 & \\
\hline $4 a-4 b$ & 66 & 34 & 32 & \\
\hline Lymph node metastasis & & & & 0.813 \\
\hline No & 120 & 59 & 61 & \\
\hline N1 & 30 & 14 & 16 & \\
\hline $\mathrm{N} 2$ & 33 & 19 & 14 & \\
\hline N3 & 35 & 17 & 18 & \\
\hline pTNM stage & & & & 0.963 \\
\hline $\mathrm{Ia}-\mathrm{Ib}$ & 92 & 45 & 47 & \\
\hline IIa-IIb & 51 & 26 & 25 & \\
\hline IIIa-IIIc & 75 & 38 & 37 & \\
\hline Operation time (min) & & $399(177-911)$ & $392(218-836)$ & 0.466 \\
\hline Intraoperative bleeding $(\mathrm{ml})$ & & $100(0-5850)$ & $90(0-3600)$ & 0.577 \\
\hline Albumin (g/dl) & & $3.4(1.5-4.0)$ & $4.2(3.5-5.1)$ & $<0.001$ \\
\hline $\mathrm{CRP}(\mathrm{mg} / \mathrm{l})$ & & $0.22(0.01-7.09)$ & $0.08(0.01-3.30)$ & $<0.001$ \\
\hline CEA (ng/ml) & & & & 0.204 \\
\hline$<5$ & 166 & 79 & 87 & \\
\hline$\geq 5$ & 52 & 30 & 22 & \\
\hline Adjuvant chemotherapy & & & & 0.035 \\
\hline Yes & 79 & 32 & 47 & \\
\hline No & 139 & 77 & 62 & \\
\hline
\end{tabular}

PNI: Prognostic nutritional index; BMI: body mass index; WBC: white blood cell; RBC: red blood cell; EGJ: esophagogastric junction; U: upper; M: middle; L: lower; Differentiation: tumor differentiation; LTG: laparoscopic total gastrectomy; LPG: laparoscopic proximal gastrectomy; L(A)DG: laparoscopic (assisted) distal gastrectomy; pTNM stage: pathological TNM stage; CRP: C-reactive protein; CEA: carcinoembryonic antigen.

$p<0.001)$, CEA level $(\mathrm{HR}=1.817,95 \% \mathrm{CI}=1.068-3.023$; $p=0.028)$, and the PNI $(\mathrm{HR}=3.289,95 \% \mathrm{CI}=1.869-6.003$; $p<0.001)$ were confirmed as independent prognostic factors for OS in PSM patients (Table III).
In the univariate analysis, a large tumor size $(p=0.025)$, advanced pTNM stage $(p<0.001)$, a low PNI value $(p=0.049)$, a high serum CEA level $(p=0.040)$, and adjuvant chemotherapy $(p=0.008)$ were significantly associated with CSS. In the 


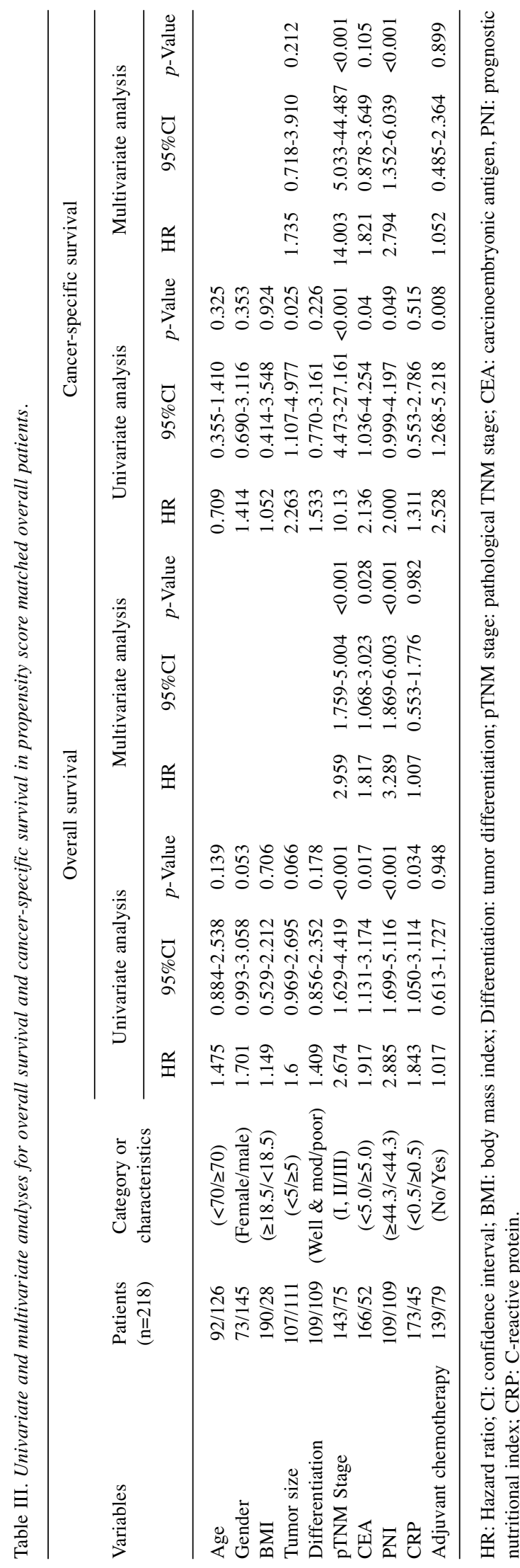

multivariate analysis, pTNM stage $(\mathrm{HR}=14.003,95 \% \mathrm{CI}=5.033$ $44.487 ; p<0.001)$ and the PNI $(\mathrm{HR}=2.794,95 \% \mathrm{CI}=1.352-$ $6.039 ; p<0.001)$ were confirmed as independent prognostic factors for CSS in PSM patients (Table III).

Postoperative survival analysis stratified by the PNI in the entire cohort of propensity score-matched patients with gastric cancer. In the PSM dataset, Kaplan-Meier analysis and the log-rank test demonstrated that patients with a low PNI value had a significantly poorer prognosis in terms of OS and CSS than those with a high PNI value ( $p<0.001$ and $p=0.008$, respectively). The 5 -year OS and CSS rates for patients with a low and high PNI value were $50.9 \%$ and $80.4 \%$ and $73.6 \%$ and $84.2 \%$, respectively (Figure 3a-b).

Clinicopathological characteristics after applying propensity score matched analysis in age-stratified patients. After applying PSM analysis, tumor depth, lymph node metastasis, and pTNM stage were not significantly different between the low and high PNI groups in age-stratified patients. Finally, 92 non-elderly patients $(42.2 \%)$ and 126 elderly patients $(57.8 \%)$ were selected for analysis.

PNI values were significantly associated with age $(p=0.004)$, BMI $(p=0.046)$, white blood cell count $(p=0.017)$, red blood cell count $(p<0.001)$, tumor size $(p=0.016)$, serum albumin concentration $(p<0.001)$, and CRP level $(p<0.001)$ in the 92 non-elderly patients with gastric cancer (Table IV).

Conversely, PNI values were significantly associated with age $(p=0.024)$, red blood cell count $(p<0.001)$, serum albumin concentration $(p<0.001)$, and CRP levels $(p=0.012)$ in the 126 elderly patients with gastric cancer (Table IV).

Cox regression analysis of postoperative survival in nonelderly patients. In the 92 non-elderly patients, univariate analysis identified advanced pTNM stage $(p<0.001)$ and a low PNI value $(p<0.001)$ as significant risk factors for shorter OS. In the multivariate analysis, pTNM stage $(\mathrm{HR}=10.784,95 \% \mathrm{CI}=4.014-33.398 ; p<0.001)$ and the PNI $(\mathrm{HR}=6.178,95 \% \mathrm{CI}=2.353-17.905 ; p<0.001)$ were confirmed as independent prognostic factors for OS (Table V).

Univariate analysis identified a large tumor size $(p=0.041)$, advanced pTNM stage $(p<0.001)$, a low PNI value $(p=0.011)$, and adjuvant chemotherapy $(p=0.018)$ as significant risk factors for shorter CSS. In the multivariate analysis, pTNM stage $(\mathrm{HR}=145.018,95 \% \mathrm{CI}=20.010$ $1,566.193 ; p<0.001)$, the PNI $(\mathrm{HR}=4.751,95 \% \mathrm{CI}=1.571$ $16.442 ; p<0.001)$, and adjuvant chemotherapy (HR=6.310, $95 \% \mathrm{CI}=1.214-33.210 ; p=0.029)$ were confirmed as independent prognostic factors for CSS (Table V).

Cox regression analysis of postoperative survival in elderly patients. In the 126 elderly patients, univariate analysis identified a low PNI value $(\mathrm{HR}=2.234,95 \% \mathrm{CI}=1.138-4.790$; 
(a)

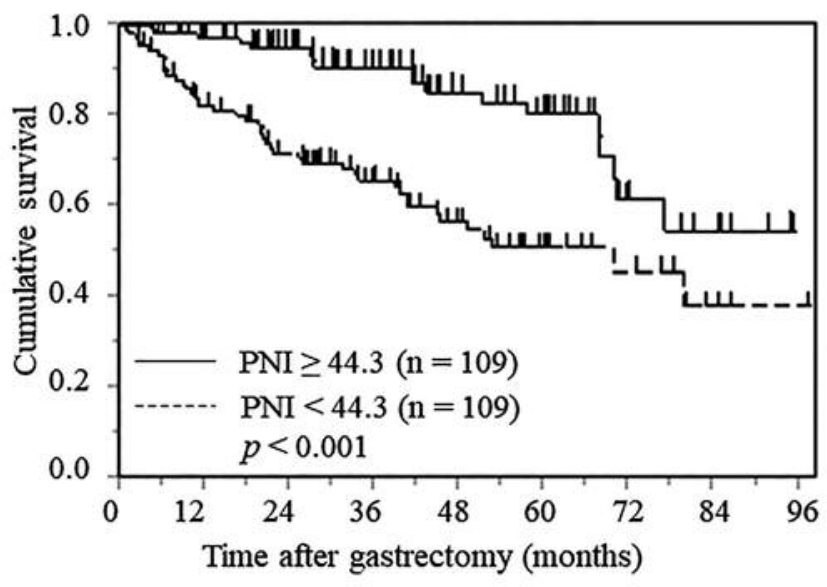

(b)

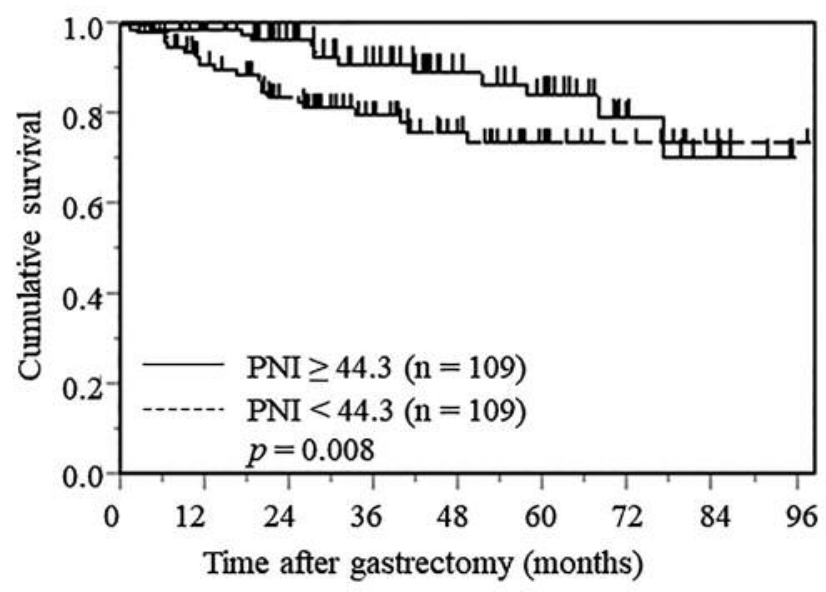

Figure 3. Kaplan-Meier curves of postoperative survival. (a) Overall survival and (b) cancer-specific survival according to the prognostic nutritional index (PNI) values in the propensity score-matched analysis of all patients.

$p=0.019)$ as a significant risk factor for shorter OS and advanced pTNM stage $(\mathrm{HR}=5.839,95 \% \mathrm{CI}=2.032-20.903$; $p<0.001)$ as a significant risk factor for shorter CSS (Table VI).

Postoperative survival analysis stratified by the PNI in nonelderly patients with gastric cancer. Kaplan-Meier analysis and the log-rank test demonstrated that patients with a low PNI value had a significantly poorer prognosis in terms of OS and CSS than those with a high PNI value $(p<0.001$ and $p=0.003$, respectively). The 5 -year OS and CSS rates for patients with a low and high PNI value were $46.6 \%$ and $88.1 \%$ and $54.7 \%$ and $85.7 \%$, respectively (Figure $4 a-b)$.

Postoperative survival analysis stratified by the PNI in elderly patients with gastric cancer. Kaplan-Meier analysis and the log-rank test demonstrated that patients with a low PNI value had a significantly poorer prognosis in terms of OS, but not CSS, than those with a high PNI value ( $p=0.015$ and $p=0.250$, respectively). The 5-year OS and CSS rates for patients with a low and high PNI value were $52.5 \%$ and $71.5 \%$ and $82.5 \%$ and $82.3 \%$, respectively (Figure 5a-b).

Age-stratified PNI values in 218 propensity score matched patients with gastric cancer. In the age-stratified analysis, the PNI value was significantly lower in elderly patients than in non-elderly patients $(p<0.001)$. The mean PNI values for non-elderly and elderly patients were $47.97 \pm 8.31$ and $42.95 \pm 6.56$, respectively (Figure 6).

\section{Discussion}

There is growing concern regarding prognostic factors for accurately identifying cancer patients at high-risk of recurrence or metastasis, which may allow us to successfully manage these patients and improve survival (10-12). Recent evidence suggests that long-term survival in patients with cancer is determined not only by tumor-related factors, but also by host-related factors. In particular, nutritional status, cancer-related inflammation, and several nutritional-based indices, including the PNI, Controlling Nutritional Status, BMI, Glasgow Prognostic Score, and preoperative body weight loss, have proved to have important prognostic value in various types of malignancies, including gastrointestinal cancer (13-15). The PNI, which is based on serum albumin and absolute peripheral lymphocyte count, was originally used to predict perioperative immuno-nutritional status and postoperative complications after gastrointestinal surgery. The PNI is simply calculated and is easily implemented through the following scoring system: $>50$ (good nutrition), 45-50 (mild malnutrition), 40-45 (moderate malnutrition), and $<40$ (severe malnutrition) (7). Serum albumin is produced by hepatocytes and is regulated by inflammatory cytokines, including interleukin-6, tumor necrosis factoralpha, and oxidative stress, which are surrogate biomarkers of the more aggressive behavior of the tumor. These proinflammatory cytokines are produced by the tumor itself or the host and play a crucial role in carcinogenesis, cancer progression, and angiogenesis (16-18). Therefore, low albumin levels may lead to impaired immune function and reduced response to treatment in patients with cancer. Lymphocytes play a critical role in the antitumor effect by initiating cytotoxic immune responses. Therefore, lymphocytopenia may serve as an indicator of an impaired tumor-associated immune system and thus promote tumor progression (19-20). However, few studies have evaluated the impact of preoperative nutritional status on long-term 
Table IV. Baseline characteristics of age stratified propensity matched gastric cancer patients.

\begin{tabular}{|c|c|c|c|c|c|c|c|c|}
\hline \multirow[b]{3}{*}{ Characteristics } & \multicolumn{4}{|c|}{ Non-elderly patients } & \multicolumn{4}{|c|}{ Elderly patients } \\
\hline & \multicolumn{5}{|c|}{ PNI } & \multicolumn{3}{|c|}{ PNI } \\
\hline & $\begin{array}{c}\text { Total } \\
\text { patients }\end{array}$ & $\begin{array}{l}<44.3 \\
(\mathrm{n}=32)\end{array}$ & $\begin{array}{l}\geq 44.3 \\
(\mathrm{n}=60)\end{array}$ & $p$-Value & $\begin{array}{c}\text { Total } \\
\text { patients }\end{array}$ & $\begin{array}{l}<44.3 \\
(\mathrm{n}=77)\end{array}$ & $\begin{array}{l}\geq 44.3 \\
(\mathrm{n}=49)\end{array}$ & $p$-Value \\
\hline Age (years) & & $65(46-69)$ & $60(36-69)$ & 0.004 & & $81(70-91)$ & $78(70-89)$ & 0.024 \\
\hline Gender & & & & 0.503 & & & & 0.069 \\
\hline Male & 62 & 23 & 39 & & 83 & 46 & 37 & \\
\hline Female & 30 & 9 & 21 & & 43 & 31 & 12 & \\
\hline BMI & & $21(13.9-29.8)$ & $22.3(16.8-32.8)$ & 0.046 & & $21.6(14.9-30.7)$ & $22.9(17.9-28.4)$ & 0.079 \\
\hline WBC $(\mu \mathrm{l})$ & & $5290(1830-9380) 60$ & $6095(3160-9280)$ & 0.017 & & $5320(2880-9750)$ & $5550(3080-10300)$ & 0.269 \\
\hline $\mathrm{RBC}\left(\times 10^{4} \mu \mathrm{l}\right)$ & & $376.5(248-484)$ & $446.5(328-548)$ & $<0.001$ & & $365(223-520)$ & $398(279-536)$ & $<0.001$ \\
\hline Location of tumor & & & & 0.3669 & & & & 0.255 \\
\hline EGJ & 2 & 0 & 2 & & 4 & 2 & 2 & \\
\hline $\mathrm{U}$ & 16 & 8 & 8 & & 25 & 15 & 10 & \\
\hline M & 43 & 15 & 28 & & 48 & 25 & 23 & \\
\hline $\mathrm{L}$ & 31 & 9 & 22 & & 49 & 35 & 14 & \\
\hline Tumor size (mm) & & $51(13-120)$ & $35(8-170)$ & 0.016 & & $58(7-170)$ & $52(5-180)$ & 0.158 \\
\hline Procedure & & & & 0.140 & & & & 0.440 \\
\hline LTG & 24 & 12 & 12 & & 36 & 19 & 17 & \\
\hline LPG & 7 & 3 & 4 & & 7 & 4 & 3 & \\
\hline $\mathrm{L}(\mathrm{A}) \mathrm{DG}$ & 61 & 17 & 44 & & 83 & 54 & 29 & \\
\hline Differentiation & & & & 0.201 & & & & 0.442 \\
\hline Well & 10 & 6 & 4 & & 17 & 8 & 9 & \\
\hline Moderate & 30 & 9 & 21 & & 52 & 33 & 19 & \\
\hline Poor & 52 & 17 & 35 & & 57 & 36 & 21 & \\
\hline Depth of tumor & & & & 0.270 & & & & 0.663 \\
\hline T1a-1b & 37 & 12 & 25 & & 43 & 28 & 15 & \\
\hline 2 & 13 & 2 & 11 & & 14 & 10 & 4 & \\
\hline 3 & 14 & 5 & 9 & & 31 & 18 & 13 & \\
\hline $4 a-4 b$ & 28 & 13 & 15 & & 38 & 21 & 17 & \\
\hline \multicolumn{9}{|l|}{ Lymph node } \\
\hline metastasis & & & & 0.489 & & & & 0.893 \\
\hline No & 53 & 17 & 36 & & 67 & 42 & 25 & \\
\hline N1 & 10 & 2 & 8 & & 20 & 12 & 8 & \\
\hline $\mathrm{N} 2$ & 11 & 5 & 6 & & 22 & 14 & 8 & \\
\hline N3 & 18 & 8 & 10 & & 17 & 9 & 8 & \\
\hline Pathological stage & & & & 0.484 & & & & 0.590 \\
\hline $\mathrm{Ia}-\mathrm{Ib}$ & 45 & 14 & 31 & & 47 & 31 & 16 & \\
\hline IIa-IIb & 17 & 5 & 12 & & 34 & 21 & 13 & \\
\hline IIIa-IIIc & 30 & 13 & 17 & & 45 & 225 & 20 & \\
\hline Operation time (min) & & $416(177-911)$ & $389(275-836)$ & 0.974 & & $389(204-714)$ & $392(218-701)$ & 0.501 \\
\hline \multicolumn{9}{|l|}{ Intraoperative blood } \\
\hline loss (ml) $55(0-2600)$ & & $50(0-2230)$ & & 0.916 & & $120(0-5850)$ & $100(00-3600)$ & 0.954 \\
\hline Albumin $(\mathrm{g} / \mathrm{dl})$ & & $3.4(1.5-3.9)$ & $4.3(3.6-5.1)$ & $<0.001$ & & $3.4(1.7-4.0)$ & $4.0(3.5-4.9)$ & $<0.001$ \\
\hline $\mathrm{CRP}(\mathrm{mg} / \mathrm{l})$ & & $0.275(0.01-7.09)$ & 9) $0.06(0.01-1.03)$ & $<0.001$ & & $0.22(0.01-6.25)$ & $0.10(0.01-3.30)$ & 0.012 \\
\hline CEA (ng/ml) & & & & 0.961 & & & & 0.287 \\
\hline$<5$ & 75 & 26 & 49 & & 91 & 53 & 38 & \\
\hline$\geq 5$ & 17 & 6 & 11 & & 35 & 24 & 11 & \\
\hline \multicolumn{9}{|l|}{ Adjuvant } \\
\hline chemotherapy & & & 0.789 & & & & & 0.064 \\
\hline Yes & 42 & 14 & 28 & & 37 & 18 & 19 & \\
\hline No & 50 & 18 & 32 & & 89 & 59 & 30 & \\
\hline
\end{tabular}

PNI: Prognostic nutritional index; BMI: body mass index; WBC: white blood cell; RBC: red blood cell; EGJ: esophagogastric junction; U: upper; M: middle; L: lower; Differentiation: tumor differentiation; LTG: laparoscopic total gastrectomy; LPG: laparoscopic proximal gastrectomy; L(A)DG: laparoscopic (assisted) distal gastrectomy; pTNM stage: pathological TNM stage; CRP: C-reactive protein; CEA: carcinoembryonic antigen. 

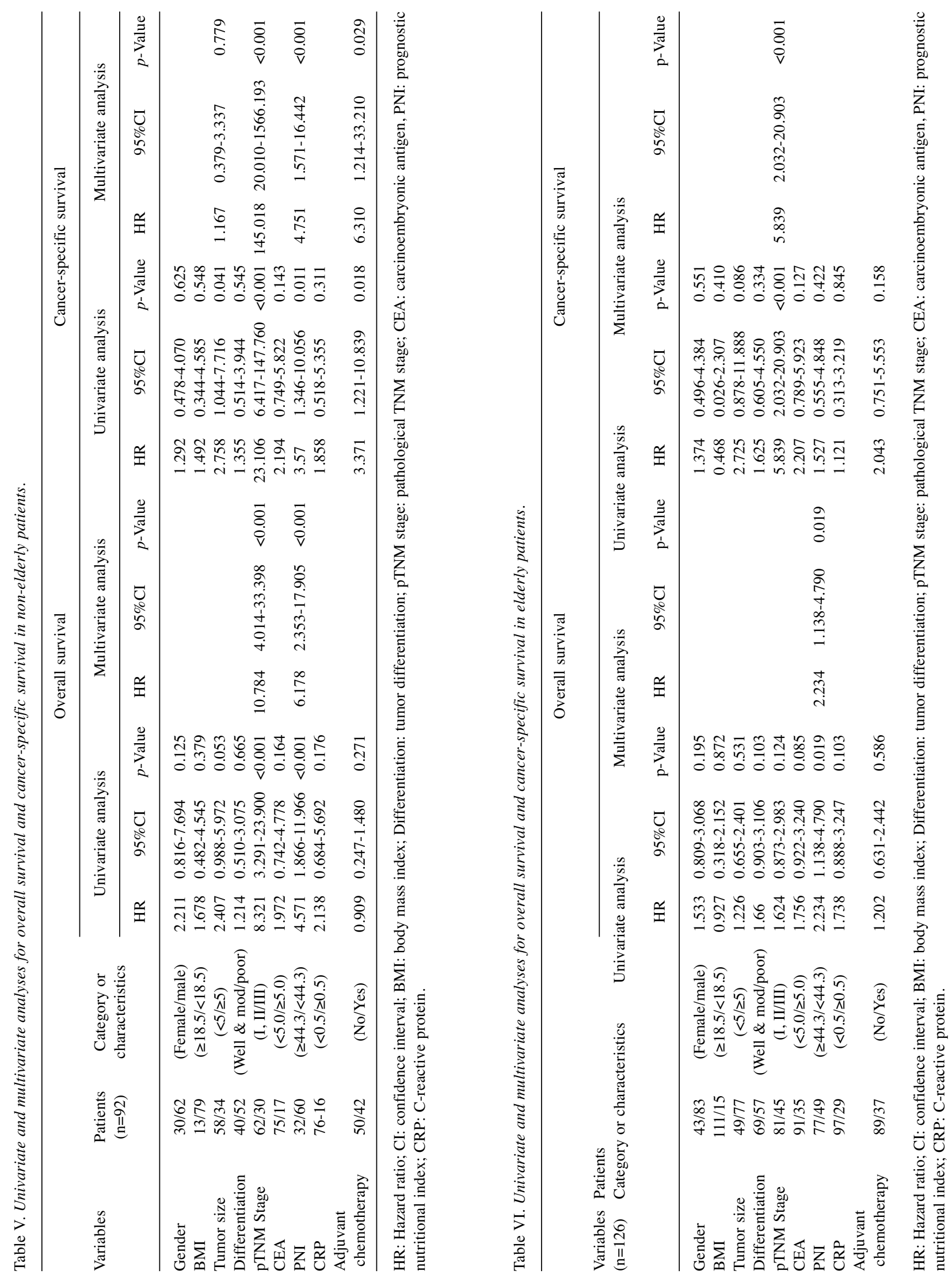
(a)

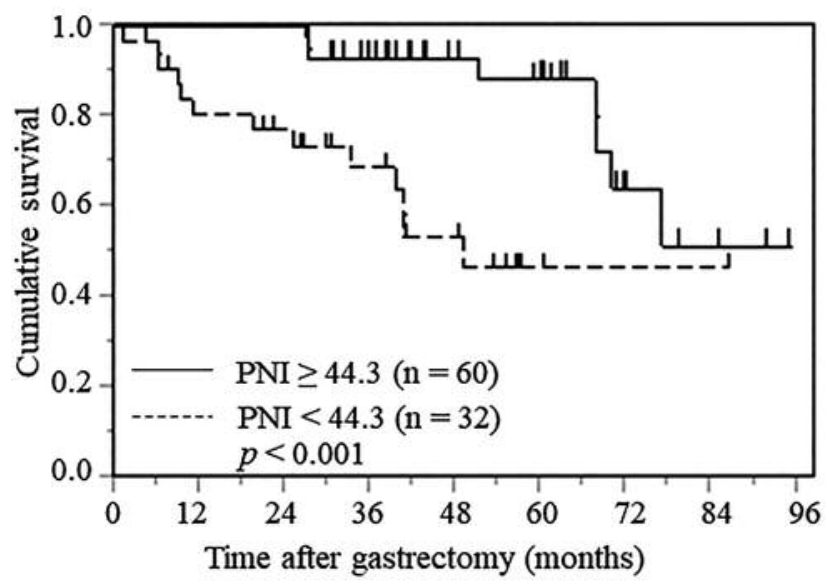

(b)

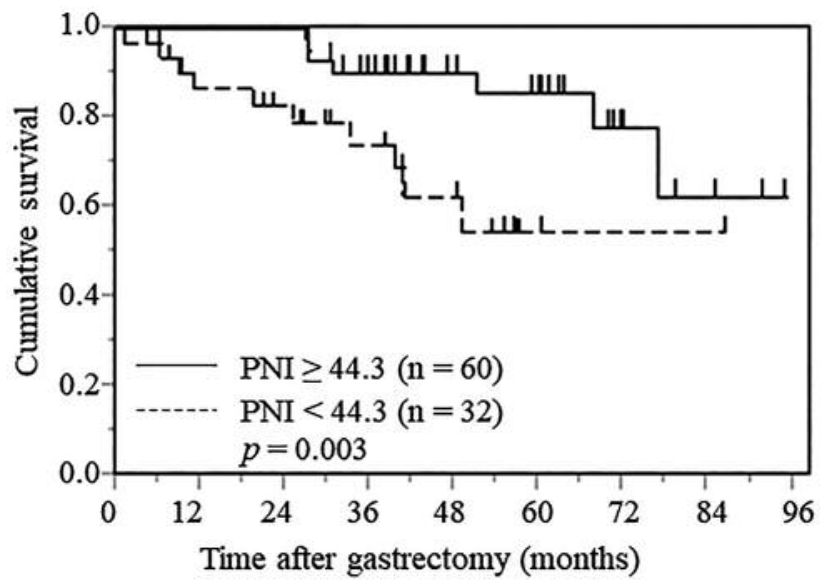

Figure 4. Kaplan-Meier curves of postoperative survival. (a) Overall survival and (b) cancer-specific survival according to the prognostic nutritional index (PNI) values in the propensity score-matched analysis of non-elderly patients.

(a)

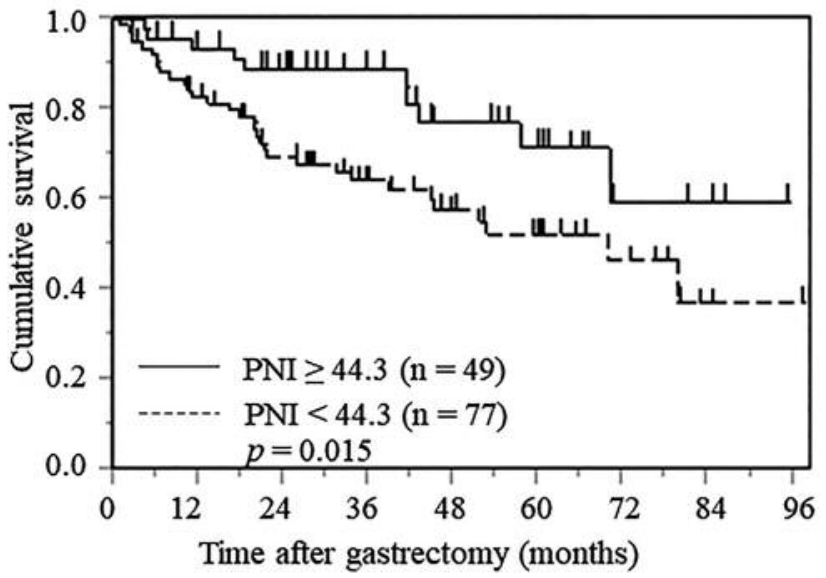

(b)

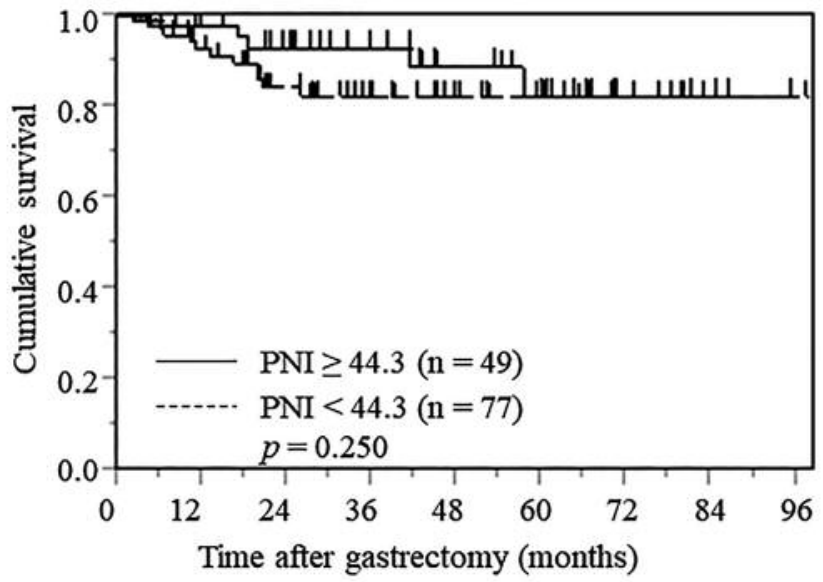

Figure 5. Kaplan-Meier curves of postoperative survival. (a) Overall survival and (b) cancer-specific survival according to the prognostic nutritional index (PNI) values in the propensity score-matched analysis of elderly patients.

survival after gastrectomy for gastric cancer. In this study, PSM analysis was used to determine the ability of the PNI to predict long-term survival in patients with gastric cancer who underwent curative gastrectomy.

The optimal cut-off value of the PNI for predicting postoperative survival in patients with malignant tumors remains controversial and a universal cutoff value has not yet been established $(4-6,21)$. Therefore, we aimed to evaluate the prognostic significance of the PNI, as well as to determine an optimal cutoff value, which can better predict OS and CSS in patients with gastric cancer. ROC analysis was performed and an optimal cutoff value for the preoperative PNI was set at 44.3 based on OS and CSS. Using this cut-off value, significant associations between a low PNI value and hostand tumor-related factors, such as advanced age, a low BMI, a deeper tumor depth, lymph node metastasis, and a more advanced pTNM stage, were confirmed in this study. In addition, PSM analysis was performed to reduce bias due to different distributions of covariables (tumor depth, lymph node metastasis, and pTNM stage) between groups of patients with a low and high PNI. In the multivariate analysis, the PNI, as well as the pTNM stage, were confirmed as 


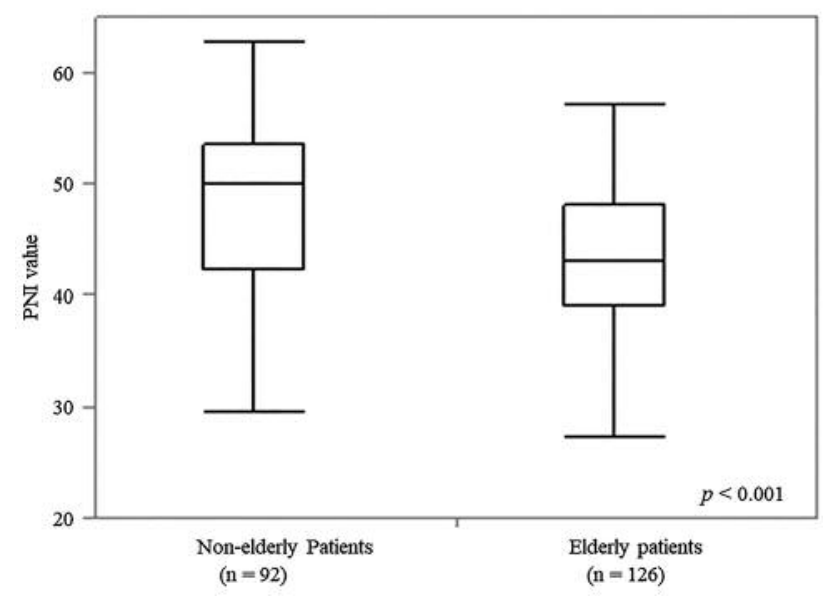

Figure 6. Box plots of the prognostic nutritional index (PNI) values according to age. In each plot, the lower and upper ends of the box represent the 25th and 75 th percentiles, respectively. Capped bars represent the minimum and maximum values. The horizontal line inside the box represents the median PNI value.

independent prognostic factors for OS and CSS in patients with gastric cancer. These findings suggest that the preoperative immune and nutritional status may be a useful surrogate marker for predicting the long-term survival of patients with gastric cancer.

In our study, the mean age of patients was 70.6 years. Previous reports have defined elderly patients as aged $\geq 70$ years (22-24). We therefore defined elderly patients as aged $\geq 70$ years. Our study revealed that a low PNI value was an independent prognostic factor for OS and CSS in the entire cohort of patients with gastric cancer. Furthermore, a low PNI value was associated with a poorer prognosis in terms of OS, but not CSS, in elderly patients with gastric cancer. Specifically, patients with a low PNI value were more likely to die of diseases other than gastric cancer than patients with a high PNI value. Additionally, our study showed that the PNI value was significantly lower in elderly patients than in non-elderly patients with gastric cancer. These findings suggest that the PNI may be a non-specific marker of tumor progression since hypoalbuminemia and leukocytopenia are often associated with aging, irrespective of the patient having cancer (25). However, the precise mechanism(s) underlying the role of the PNI as a predictor of prognosis in patients with cancer has not yet been established.

Gastric outlet obstruction often disturbs the nutritional status of patients with advanced gastric cancer. Preoperative immuno-nutritional support through parenteral nutrition infusion with albumin may be beneficial for cancer patients with malnutrition. Recent reports suggest that immunonutritional support improves not only short-term survival, but also long-term survival in gastric cancer patients with malnutrition, although the underlying mechanism(s) remain controversial $(26,27)$.

Several limitations need to be considered when interpreting the results of this study. First, this study was uncontrolled and retrospective in nature. Second, this was a single-center study with a relatively small sample size and short follow-up durations. Third, immune and nutritional parameters, such as interleukin- 6 and tumor necrosis factoralpha, were not adequately evaluated. Finally, there was insufficient evidence regarding the validity of the cut-off values for the PNI. Therefore, further studies are needed to establish appropriate cutoff values for the PNI. Large prospective randomized controlled trials should also be conducted to confirm our preliminary findings.

Despite the above limitations, PNI was confirmed to be a potentially novel prognostic factor for predicting OS and CSS under the cut-off value of 44.3 in patients with gastric cancer who underwent curative gastrectomy, especially non-elderly patients. Because the PNI is convenient, useful, and readily available at low cost, it has the potential to serve as a biomarker for predicting the survival of patients with gastric cancer.

\section{Conflicts of Interest}

The Authors declare that they have no competing interests regarding this study.

\section{References}

1 Sobin L, Gospodarowicz M and Wittekind C (eds.): International Union Against Cancer (UICC). TNM classification of malignant tumors, 7th ed. New York: Wiley-Blackwell, 2010.

2 Hirahara N, Matsubara T, Mizota Y, Ishibashi S and Tajima Y: Prognostic value of preoperative inflammatory response biomarkers in patients with esophageal cancer who undergo a curative thoracoscopic esophagectomy. BMC Surg 16(1): 66, 2016.

3 Mimatsu K, Fukino N, Ogasawara Y, Saino Y and Oida T: Utility of inflammatory marker- and nutritional status-based prognostic factors for predicting the prognosis of stage IV gastric cancer patients undergoing non-curative surgery. Anticancer Res 37(8): 4215-4222, 2017.

4 Onodera T, Goseki N and Kosaki G: Prognostic nutritional index in gastrointestinal surgery of malnourished cancer patients [in Japanese]. Nihon Geka Gakkai Zasshi 85: 1001-1005, 1984.

5 Lin YH, Chang KP, Lin YS and Chang TS: Pretreatment combination of platelet counts and neutrophil-lymphocyte ratio predicts survival of nasopharyngeal cancer patients receiving intensity-modulated radiotherapy. Onco Targets Ther 10: 27512760, 2017.

6 Kim A, Im M and Ma JY: Sosiho tang ameliorates cachexia related symptoms in mice bearing colon 26 adenocarcinoma by reducing systemic inflammation and muscle loss. Oncol Rep 35(3): 1841-1850, 2016.

7 Dev R, Wong A, Hui D and Bruera E: The Evolving Approach to Management of Cancer Cachexia. Oncology (Williston Park) 31(1): 23-32, 2017. 
8 Wang Y, Wang Y, Mu H, Liu T, Chen X and Shen Z: Enhanced specific antitumor immunity of dendritic cells transduced with the glypican 3 gene and co-cultured with cytokine-induced killer cells against hepatocellular carcinoma cells. Mol Med Rep 11(5): 3361-3367, 2015.

9 Japanese Gastric Cancer Association: Japanese gastric cancer treatment guidelines 2010 (ver.3). Gastric Cancer 14: 113-123, 2011.

10 Hirahara N, Matsubara T, Hayashi H, Takai K, Fujii Y and Tajima Y: Impact of inflammation-based prognostic score on survival after curative thoracoscopic esophagectomy for esophageal cancer. Eur J Surg Oncol 41(10): 1308-1315, 2015.

11 Song A, Eo W and Lee S: Comparison of selected inflammationbased prognostic markers in relapsed or refractory metastatic colorectal cancer patients. World J Gastroenterol 21(43): 1241012420, 2015.

12 Chen C, Chen Q, Zhao Q, Liu M and Guo J: Value of Combined Detection of Serum CEA, CA72-4, CA19-9, CA15-3 and CA125 in the Diagnosis of Gastric Cancer. Ann Clin Lab Sci 47(3): 260-263, 2017.

13 Ohashi T, Komatsu S, Ichikawa D, Kosuga T, Okamoto K, Arita T, Konishi H, Morimura R, Murayama Y, Shiozaki A, Kuriu Y, Ikoma $\mathrm{H}$, Nakanishi M, Fujiwara $\mathrm{H}$ and Otsuji E: Monitoring with sensitive tumor markers contributes to decision-making and better prognosis in gastric cancer patients with peritoneal recurrence. Int J Clin Oncol 22(5): 897-904, 2017.

14 Shimizu K, Okita R, Saisho S, Maeda A, Nojima Y and Nakata M: Preoperative neutrophil/lymphocyte ratio and prognostic nutritional index predict survival in patients with non-small cell lung cancer. World J Surg Oncol 13: 291, 2015.

15 Okamura Y, Ashida R, Ito T, Sugiura T, Mori K and Uesaka K: Preoperative neutrophil to lymphocyte ratio and prognostic nutritional index predict overall survival after hepatectomy for hepatocellular carcinoma. World J Surg 39(6): 1501-1509, 2015.

16 Ikeguchi M, Urushibara S, Shimoda R, Yamamoto M, Maeda Y and Ashida K: Inflammation-based prognostic scores and nutritional prognostic index in patients with locally-advanced unresectable colorectal cancer. World J Surg 12: 210, 2014.

17 Balkwill F: Tumour necrosis factor and cancer. Nat Rev Cancer 9(5): 361-371, 2009.

18 Brenner D, Blaser $\mathrm{H}$ and Mak TW: Regulation of tumour necrosis factor signalling: live or let die. Nat Rev Immunol 15(6): 362-374, 2015.

19 Gupta D and Lis CG: Pretreatment serum albumin as a predictor of cancer survival: a systematic review of the epidemiological literature. Nutr J 9: 69, 2010.
20 Ray-Coquard I, Cropet C, Van Glabbeke M, Sebban C, Le Cesne A, Judson I, Tredan O, Verweij J, Biron P, Labidi I, Guastalla JP, Bachelot T, Perol D, Chabaud S, Hogendoorn PC, Cassier P, Dufresne A and Blay JY; European Organization for Research and Treatment of Cancer Soft Tissue and Bone Sarcoma Group: Lymphopenia as a prognostic factor for overall survival in advanced carcinomas, sarcomas, and lymphomas. Cancer Res 69(13): 5383-5391, 2009.

21 Aoyama T, Murakawa M, Katayama Y, Yamaoku K, Kanazawa A, Higuchi A, Shiozawa M, Morimoto M, Yoshikawa T, Yamamoto N, Rino Y, Masuda M and Morinaga S: Impact of postoperative complications on survival and recurrence in pancreatic cancer. Anticancer Res 35(4): 2401-2409, 2015.

22 Kulu Y, Tarantio I, Warschkow R, Kny S, Schneider M, Schmied $\mathrm{BM}$, Büchler MW and Ulrich A: Anastomotic leakage is associated with impaired overall and disease-free survival after curative rectal cancer resction: a propensity score analysis. Ann Surg Oncol 22(6): 2059-2067, 2015.

23 Tokunaga M, Tanizawa Y, Bando E, Kawamura T and Terashima M: Poor survival rate in patients with postoperative intraabdominal infectious complications following curative gastrectomy for gastric cancer. Ann Surg Oncol 20(5): 15751583, 2013.

24 Téllez-Avila FI and García-Osogobio SM: The carcinoembryonic antigen: apropos of an old friend. Rev Invest Clin 57(6): 814-819, 2005.

25 Wong-Chong N, Kehlet H and Grantcharov TP: Outcomes from an enhanced recovery program for laparoscopic gastric surgery. Surg Laparosc Endosc Percutan Tech 26(3): e50-55, 2016.

26 Sakurai K, Ohira M, Tamura T, Toyokawa T, Amano R, Kubo N, Tanaka H, Muguruma K, Yashiro M, Maeda K and Hirakawa $\mathrm{K}$ : Predictive potential of preoperative nutritional status in longterm outcome projections for patients with gastric cancer. Ann Surg Oncol 23(2): 525-533, 2015.

27 Miao J, Xiao W, Wang L, Han F, Wu H, Deng X, Gui X and Zhao C: The value of the Prognostic Nutritional Index (PNI) in predicting outcomes and guiding the treatment strategy of nasopharyngeal carcinoma (NPC) patients receiving intensitymodulated radiotherapy (IMRT) with or without chemotherapy. J Cancer Res Clin Oncol 143(7): 1263-1273, 2017.

Received May 23, 2018

Revised June 12, 2018

Accepted June 13, 2018 